\title{
Novel Method for 3D Objects Retrieval
}

\author{
Dayanand Jamkhandikar \\ Research Scholar \\ NIET, CS\&E Department \\ NIMS University, JAIPUR
}

\author{
Surendra Pal Singh, PhD \\ HOD CSE Dept. \\ NIMS Inst. of Engg, \& Tech. \\ NIMS University, JAIPUR
}

\author{
V.D. Mytri, PhD \\ Principal \\ Appa Institute of Engg. \& Tech.
}

\begin{abstract}
Access to appropriate information is a fundamental necessity in the modern society. Recent years have seen a rapid growth in use of digital images. To retrieve similar images from a database Content Based Image Retrieval (CBIR) system is used. Shape is most widely used feature in CBIR system. Among various approaches of shape retrieval, edge based shape descriptors are the most commonly been used. These representations are processed via different edge estimation tools and algorithms. However in the process of edge based coding, the discontinuous edge regions result in discarding of image regions or the inclusion of such regions increases the number of processing regions. A selection of non-informative region will lead to less descriptive feature, which leads to increase in system overhead and lowering of retrieval accuracy. Contours are observed to be the best representative approach for shape descriptor. To improve the retrieval accuracy of a 3D image retrieval system, a contour based coding is developed. In this approach along with the shape feature depth feature is extracted from the contor. The proposed 3D contor Shape depth (3D CSD) approach uses less number of feature vectors for representation. Simulation observations show that 3D CSD improves the retrieval accuracy and reduce the computation time.
\end{abstract}

\section{Keywords}

CBIR, 3D-CSD, shape feature, contor, edge.

\section{INTRODUCTION}

In the process of image coding, wherein image information are coded to represent into an informative detail and then processed to obtain the best match information is called as image retrieval system. As in various applications the processing data are large in count or large in representation and the available resources are very lower in count. In such resource constraint environment, the processing efficiency is decreased to very low level specially with processing delay factor. The current retrieval system is observed to achieve the estimation accuracy with the cost of higher computation or the vice versa. With the current demand these algorithms are to be enhanced with various approaches exploiting the representing feature to be simpler and descriptive for ease of searching. Though such strategies are effective in capturing low-level image features, the gap between these low-level features and high-level representation is still to be merged. The retrieval of image using generic image features such as shape has become progressively vital due to the convenience of representation as an efficient descriptor. In process to image coding for image retrieval, images are captured via imaging device such as digital cameras. These capturing devices based on their arrangements and capturing features returns a 2-Dimensional or 3-Dimensional image samples. In 2-D information's is the pixel magnitude at a given row and column value. For 3D the $3^{\text {rd }}$ parameter is the width of the image sample. While processing for such 3-D images, the shape and depth features could be informative. New techniques for modeling, digitizing and visualizing 3D shapes have led to sudden increase in the number of available 3D models on the Internet and in domainspecific databases. Retrieving the similar $3 \mathrm{D}$ objects is very essential requirement for the image retrieval system. CBIR for three dimensional (3D) images primarily used to retrieve large number of images present on internet. So, the main challenge for 3D retrieval is to extract features which are invariant of viewing angles, i.e. invariant of rotation even though they may not be visible from all the viewing angles. This has motivated for the development of 3D shape retrieval systems. Aim of the proposed method is to retrieve similar 3D objects for a given a query sample image even though the object is rotated.

\section{EXISTING METHODS}

Various experimental search engines were developed to retrieve the 3D images. It includes the Ephesus search engine at the National Research Council of Canada. The 3D model search engine at Princeton University and the 3D model retrieval system at the National Taiwan University. Furthermore, domain-specific shape models are now accessible on the Internet. Most of the content-based image retrieval (CBIR) shared between $2 \mathrm{D}$ and $3 \mathrm{D}$. This has led to many current studies focusing on the invariance of transformations (including rotation, scaling and translation) of objects, which has more to do with shapes. By taking advantages of polynomial representations in [1], 3D Zernike descriptors have been developed to describe shapes of objects. Similarly, in order to achieve transformation invariance, a graph-based shape descriptor is created in [2] to determine similarity between 3D objects. Recently impact descriptors [3] are used to capture the surrounding areas of a 3D shape. Some other techniques proposed to retrieve $3 \mathrm{D}$ objects are discussed in [4][5][6][7]. Because shape-based approaches only describe the surface of a 3D object, they tend to ignore the content inside that object. Depth based descriptors therefore have been developed as demonstrated in [8]. However, limited work has carried out in retrieving domain dependent benchmark databases. If the depth value of an image is found and used along with the shape feature in coding then retrieval accuracy may improve.

\section{PROPOSED APPROACH}

In this work a novel method which uses depth feature extracted from contor of the image along with the shape feature is used to describe the image content.

\subsection{Shape feature extraction}

A digital image consists of group of pixels arranged in matrix. Simplest mode of image representation is by using shape feature. To describe the shape of an image edges play very important role. Edge operators derive coefficients out of the bounding regions in many image representation techniques. For many years the fundamental issue in image processing 
was detecting the edge of an image. Step edges are dominantly used in the detection. There are various forms of 1-D step edge operations investigated and one such approach is defined by Canny $[9,10]$. After finding the edge of the image, contour can be derived from it. For the detection of contour all the real corners must be detected. The system should avoid detecting the false corners. The contour evaluator must be effective in finding the true edges. For finding the contour, 8-region neighborhood-growing algorithm is used [11].

To extract a shape feature for a given image sample, the process is as outlined;

1. If input image is color then convert it into gray scale image.

2. Now the gray scale image is binarized. A pixel of the image can either have a value of 1 or 0 .

3. Edges from the binary image can be obtained by separating the object information from its background details.

8-point connectivity technique is applied to find Shape signature. This is a one dimensional representation of the shape, which is obtained by applying on the $2 \mathrm{D}$ closed edge region. As objects have well defined centroid $\left(\mathrm{x}_{\mathrm{c}}, \mathrm{y}_{\mathrm{c}}\right)$ and also retrieval has shown to be better with central distance, is used as a shape representation. The feature vector representing the central distance between point on the contour $(\mathrm{x}, \mathrm{y})$ and the centroid $\left(\mathrm{x}_{\mathrm{c}}, \mathrm{y}_{\mathrm{c}}\right)$ is given by:

$$
V_{c}=\left(\mathbf{x}-x_{c}, \mathrm{y}-y_{c}, 0\right)
$$

Where,

$$
x_{c}=\frac{1}{N} \sum_{i=0}^{N-1}\left(x_{i}\right)
$$

and

$$
y_{c}=\frac{1}{N} \sum_{i=0}^{N-1}\left(y_{i}\right)
$$

Where, $\mathrm{N}$ is the total number of pixels

\subsection{Extraction of depth map from contour}

In order to extract depth map, first the contour is obtained by using 8 region connectivity algorithm [11]. From this contor $3 \mathrm{D}$ information is computed. Recovering the 3D information can be done in terms of depth $\mathrm{Z}$, the surface normal $\left(\mathrm{n}_{\mathrm{x}}, \mathrm{n}_{\mathrm{y}}\right.$, $\mathrm{n}_{\mathrm{z}}$ ), or surface gradient $(\mathrm{p}, \mathrm{q})$. A principle of shape from shading is used to obtain the $3 \mathrm{D}$ embedding information. Lamberdian model, where it is assumed that equal amount of light is reflected in every direction is considered. In this model, the reflectance map is simplified to be independent of viewer's direction. To identify the depth-map (sometimes called as simply depth) of an image this work uses an approach proposed by $\mathrm{T}$ sai [12]. This approach assumes that the lower order components in the reflectance map dominate. The linearity of the reflectance map in the depth $\mathrm{Z}$ has been used instead of in $p$ and q. discrete approximations for $p$ and $q$ are employed and linearized the reflectance in $\mathrm{Z}(\mathrm{x}, \mathrm{y})$. The following equations [12] are used to find the depth of an image. The reflectance function for the Lamberdian surface is as follows:

$\mathrm{E}(\mathrm{x}, \mathrm{y})=\mathrm{R}(\mathrm{p}, \mathrm{q})$

$$
=\frac{1+p p_{s}+q q_{s}}{\left(\sqrt{1+p^{2}+q^{2}}\right) \sqrt{1+p_{s}^{2}+q_{s}^{2}}}
$$

$$
\begin{aligned}
& \mathrm{p}=\frac{\partial z}{\partial x}, \mathrm{q}=\frac{\partial z}{\partial y} \\
& p_{s}=\frac{\cos \tau \sin \sigma}{\cos \sigma} \\
& q_{s}=\frac{\sin \tau \sin \sigma}{\cos \sigma}
\end{aligned}
$$

' $\tau$ ' is the tilt of the illuminant and $\sigma$ is the slant of the illuminant. Discrete approximation of $\mathrm{p}$ and $\mathrm{q}$ are given by the following:

$$
\begin{gathered}
\mathrm{p}=\frac{\partial z}{\partial x}=\mathrm{Z}(\mathrm{x}, \mathrm{y})-\mathrm{Z}(\mathrm{x}-1, \mathrm{y}) \\
\mathrm{q}=\frac{\partial z}{\partial y}=\mathrm{Z}(\mathrm{x}, \mathrm{y})-\mathrm{Z}(\mathrm{x}, \mathrm{y}-1)
\end{gathered}
$$

The reflectance equation can be then rewritten as:

$$
\begin{gathered}
\mathrm{f}(\mathrm{E}(\mathrm{x}, \mathrm{y}), \mathrm{Z}(\mathrm{x}, \mathrm{y}), \mathrm{Z}(\mathrm{x}-1, \mathrm{y}), \mathrm{Z}(\mathrm{x}, \mathrm{y}-1)) \\
=\mathrm{E}(\mathrm{x}, \mathrm{y})-\mathrm{R}(\mathrm{Z}(\mathrm{x}, \mathrm{y})-\mathrm{Z}(\mathrm{x}-1, \mathrm{y}), \mathrm{Z}(\mathrm{x}, \mathrm{y})-\mathrm{Z}(\mathrm{x}, \mathrm{y}-1))
\end{gathered}
$$

For a given image $\mathrm{E}$ and its fixed point $(\mathrm{x}, \mathrm{y})$ linear approximation (Taylor series expansion up through the first order terms) is carried out for the function ' $\mathrm{f}$ ' about a given depth map $Z^{n-1}$. By solving using iterative Jacobi method it results in the following reduced form

$\mathrm{f}(\mathrm{Z}(\mathrm{x}, \mathrm{y}))=$

$\mathrm{f}\left(Z^{n-1}(x, y)\right)+\left(Z(x, y)-Z^{n-1}(x, y)\right) \frac{d f\left(Z^{n-1}(x, y)\right)}{d Z(x, y)}$

For $Z(x, y)=Z^{n}(x, y)$, the depth map at $\mathrm{n}^{\text {th }}$ iteration can be solved using the following:

$$
\left.Z^{n}(x, y)=Z^{n-1}(x, y)\right) \frac{d f\left(Z^{n-1}(x, y)\right)}{d Z(x, y)}
$$

Where

$$
\begin{aligned}
& \frac{d f\left(Z^{n-1}(x, y)\right)}{d Z(x, y)}= \\
& -1 *\left(\frac{p_{s}+q_{s}}{\sqrt{1+p^{2}+q^{2}} \sqrt{1+p_{s}{ }^{2}+q_{s}{ }^{2}}}-\frac{(p+q)\left(p p_{s}+q q_{s}+1\right)}{\left.\left(\sqrt{1+p^{2}+q^{2}}\right)\right)^{3} \sqrt{1+p_{s}{ }^{2}+q_{s}{ }^{2}}}\right)
\end{aligned}
$$

The depth map is then represented in a way similar to the shape. The feature vector representing depth is given by:

$$
V_{d}=\left(0,0, \mathrm{Z}-Z_{c}\right)
$$

Where $\mathrm{Z}$ is the depth obtained from Equation (12) of the contour, and $Z_{c}$ denotes the third dimension of the centroid.

Shape, in this work, is combined with the corresponding estimated depth profile. Shape-Depth can be defined as, I : R2 $\rightarrow$ R3 at each point on the contour, a vector is defined as follows:

$$
\mathrm{V}=\left(\mathrm{x}-x_{c}, \mathrm{y}-y_{c}, \mathrm{Z}-Z_{c}\right)
$$

This feature is of dimension three, which is quite low and hence it enhances the speed of retrieving. This can be decomposed into $V_{c}$ representing the shape/contour and $V_{d}$ representing depth. A weighted combination of the magnitude of the vectors $V_{c}$ and $V_{d}$ is used for retrieving images. The 3D-CSD (Contour Shape depth) representation is defined as follows:

Where $\mathrm{E}(\mathrm{x}, \mathrm{y})$ is the gray level at pixel $(\mathrm{x}, \mathrm{y})$, 


$$
S D=\frac{w_{c} *\left\|V_{c}\right\|+W_{d} *\left\|V_{d}\right\|}{W_{c}+W_{d}}
$$

Where $\mathrm{w}_{\mathrm{c}}$ represents the weights assigned to the shape-based similarity and $\mathrm{w}_{\mathrm{d}}$ the weight assigned to the depth-based similarity.

The value of $\mathrm{w}_{\mathrm{c}}+\mathrm{w}_{\mathrm{d}}=1$, for $\mathrm{w}_{\mathrm{c}}>0$ and $\mathrm{w}_{\mathrm{d}}>0$. It can be observed that $\mathrm{V}_{\mathrm{c}}$ captures the central distance measured in the 2D domain and $\mathrm{V}_{\mathrm{d}}$ is a similar measure on the third dimension, the depth. To achieve the property of invariant to transformations such as translation, scaling and rotation, Fourier transform is widely used. This transformation is used in this work for achieving the invariance. For any 1-D signature function, its discrete Fourier transform is given by:

$a_{n}=\frac{1}{N} \sum_{i=0}^{N-1}(S D) e^{-\left(-\frac{j 2 \pi i t}{N}\right)}$

$$
\text { (17) }
$$

Where, $\mathrm{n}=0,1 \ldots \ldots \mathrm{N}-1$. The coefficient $\mathrm{a}_{\mathrm{n}}$ is denoted as $\mathrm{FD}_{\mathrm{n}}$. Before applying Fourier transform on the shape signature, shape is first sampled to fixed number of points. In general, objects shape and model shape can have different sizes. Consequently, the number of data points of the object and model representations will also be different. Same number of data points and its FFT for the shape boundary of objects and models must be sampled to have the matching.

The translation invariant 3D-CSD representation is described in this work. So, the corresponding FDs are also having translation invariance. To achieve rotation invariance only the magnitude information is used and the phase information is ignored. By dividing the magnitude values of the FDs with FD1 Scale normalization is achieved. The invariant feature vector used to index $\mathrm{SD}$ is then given by;

$\mathrm{f}=\frac{\left|F D_{2}\right|}{\left|F D_{1}\right|}, \mid \frac{F D_{3} \mid}{\left|F D_{1}\right|}, \ldots \ldots \ldots ., \frac{\left|F D_{N-1}\right|}{\left|F D_{1}\right|}$

Retrieval result is not a single image but a list of images ranked by their similarities with the query image since CBIR is not based on exact matching. For a model shape indexed by FD feature

$f_{m}=\left[f_{m}^{1}, f_{m}^{2}, \ldots, f_{m}^{N}\right]$ and a database indexed by feature $f_{d}=$ $\left[f_{d}^{1}, f_{d}^{2}, \ldots, f_{d}^{N}\right]$ the Euclidean distance between two feature vectors can then be used for the similarity measurement,

$\mathrm{d}=\sqrt{\sum_{i=0}^{N-1}\left|f_{m}^{i}-f_{d}^{i}\right|^{2}}$

Where, $\mathrm{N}$ is the total number of sampled points on the shape contour.

\subsection{Contour Shape-Depth (3D CSD) Algorithm}

\section{Algorithm 3D Contour Shape-Depth}

Step 1. Read each image sequentially from image database

Step 2. Apply preprocessing on each database image

Step 3. For each image determine edge feature by applying Canny edge operator

Step 4. Use eight neighborhood growing method to obtain the contour of images

Step 5. Employ Lambradian model on obtained contour and compute the shape depth features
Step 6. Store the extracted shape depth features of all images on feature vector database

Step 7. Read the query image and apply step 2 to step 5 on query image.

Step 8. Measure Euclidean distance between all stored feature vectors and query image feature vector

Step 9. Set threshold as the mean of entire distance calculated in step 8

Step 10.Compare distance between query image feature vector and feature vector database with threshold distance.

Step 11. If distance is less than threshold distance then retrieve corresponding image.

Step 12 Repeat the step 10 and step 11 until the required numbers of images are retrieved.

Step 13. Output the top retrieved images in ascending order.

\section{SIMULATION OBSERVATIONS}

To evaluate the stated work, a simulation on different test data under different orientation is carried out using MATLAB tool. For 3D image retrieval analysis in this work COIL-100 having color 3D images [13] is used. This dataset consist of 7200 training sample, with each class containing 72 oriented samples. From the above mentioned dataset a set of 5 samples from each class is taken for testing. The other samples are utilized for training. Some of the sample images from the database are as shown in below figure 1

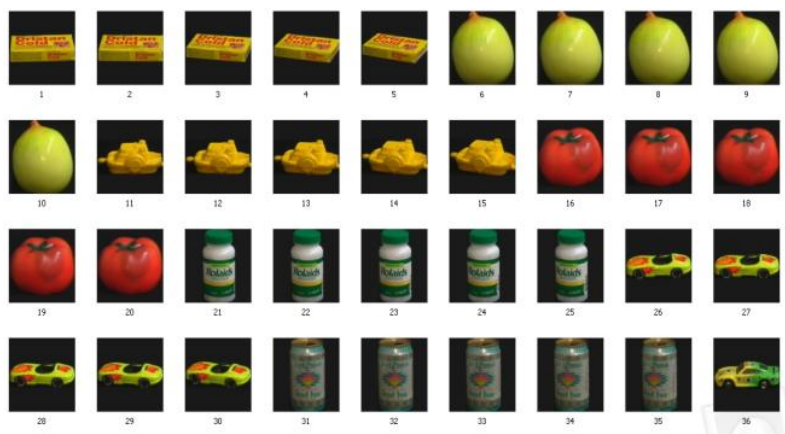

Figure 1 Sample images from database

To evaluate the process of 3D-CSD feature processing a test sample with different orientation is passed to the developed system. The same sample is also passed to the system for which the edge based features [14] are used. The obtained observations for the system is as illustrated below.

\section{a) Sample I: Sample with $0^{0}$ orientation}

Below figure 2 shows the query image. The top 4 obtained results using proposed 3D CSD approach is shown in figure 3 . Figure 4 shows top 4 samples using edge feature. Using theses extracted features, recognition process is carried out using $\mathrm{K}$ $\mathrm{NN}$ classifier 


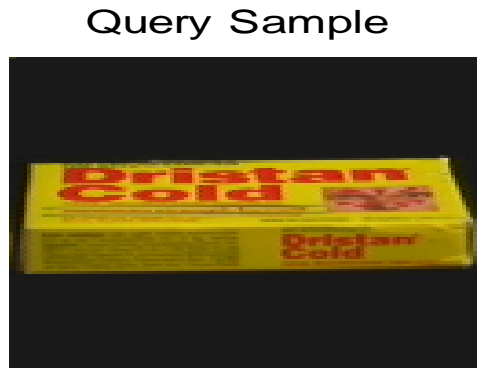

Figure 2 Query sample at $0^{0}$ orientation
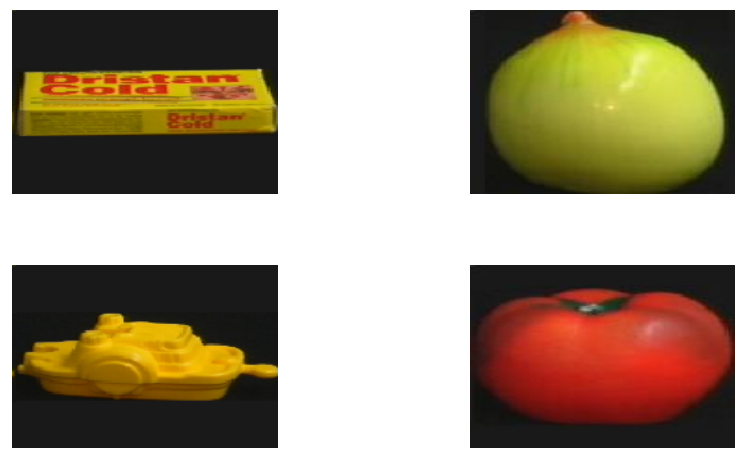

Figure 3 Top 4 samples when query is at $0^{0}$ orientation using 3D CSD approach
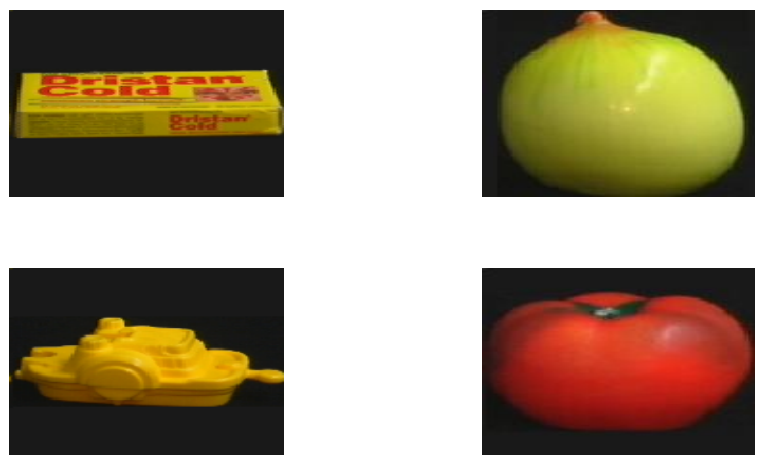

Figure 4 Top 4 samples when query is at $0^{0}$ orientation using Edge based approach

b) Sample II: Sample with $1^{\circ}$ orientation

Below figure 5 shows a query sample which is at $15^{0}$ orientations.

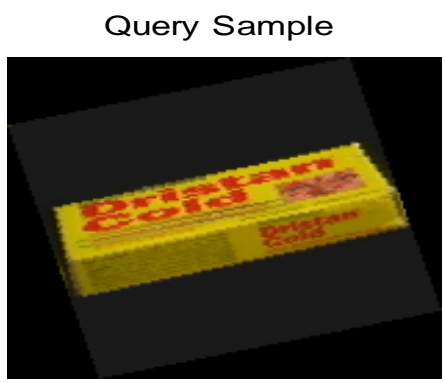

Figure 5 Query sample at $\mathbf{1 5}^{\circ}$ orientation

The obtained results using the proposed approach is shown in figure 6 and using edge based features is shown in figure 7
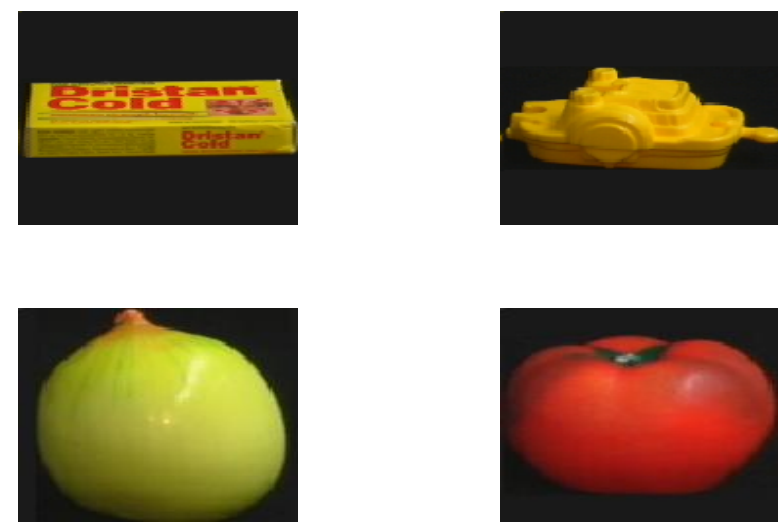

Figure 6 Top 4 samples when query is at $\mathbf{1 5}^{\circ}$ orientation using 3D CSD approach
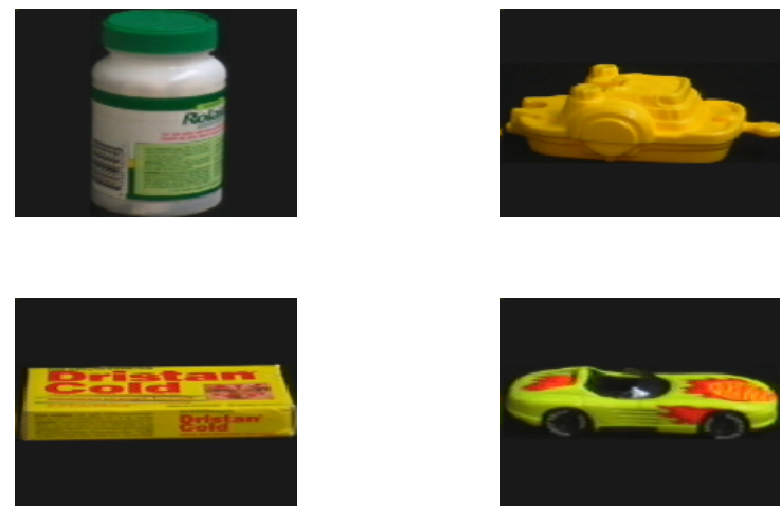

Figure 7 Top 4 samples when query is at $15^{\circ}$ orientation using Edge based approach

When applied with $\mathbf{1 5}^{\mathbf{0}}$ orientations, in proposed 3D-CSD approach in addition to the shape information, depth feature is considered, this feature reveals the orientation information, and hence these features variations are more contributive to the recognition process. However this variation is not retained in edge based shape descriptor. Hence the classification process leads to misclassification at the top of the output.

c) Sample III: Sample with $\mathbf{4 5}^{\circ}$ orientation

To test the robustness of the approach the query sample is passed at $45^{\circ}$ orientations as shown in the figure

\section{Query Sample}

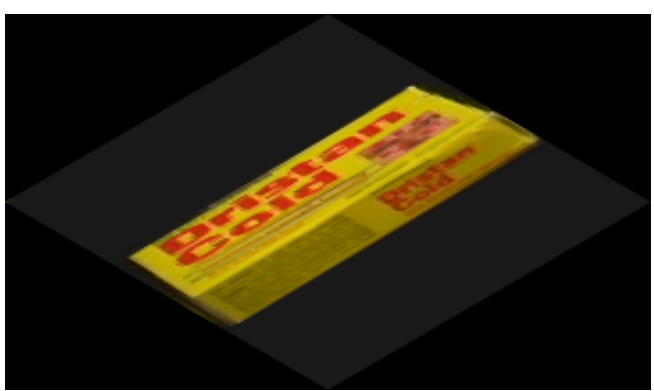

Figure 8 Query sample at $45^{\circ}$ orientation 

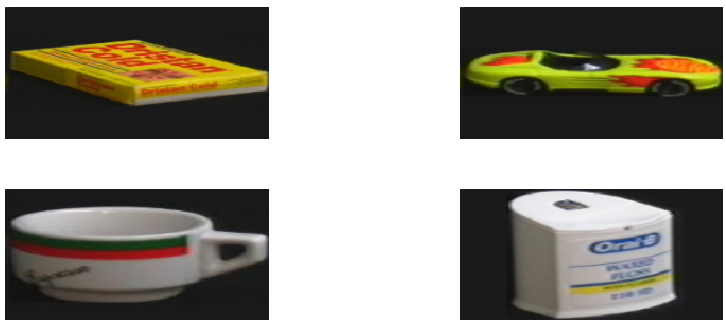

Figure 9 Top 4 samples when query is at $45^{\circ}$ orientations using 3D CSD approach
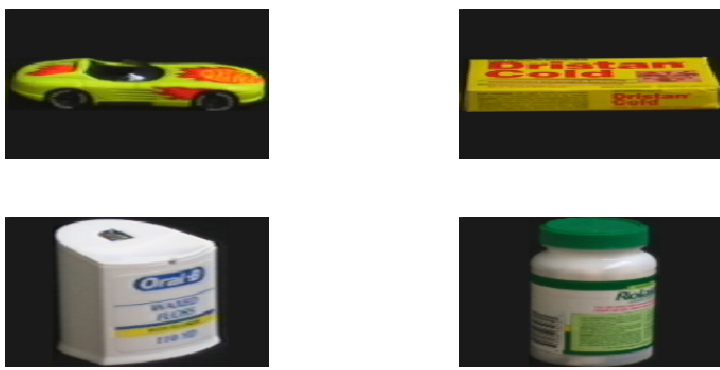

Figure 10 Top 4 samples when query is at $45^{\circ}$ orientation using Edge based approach

To observe the performance of the developed approach, an analysis is carried out for different test samples at different orientations. The test result for these samples is outlined in figure 11.

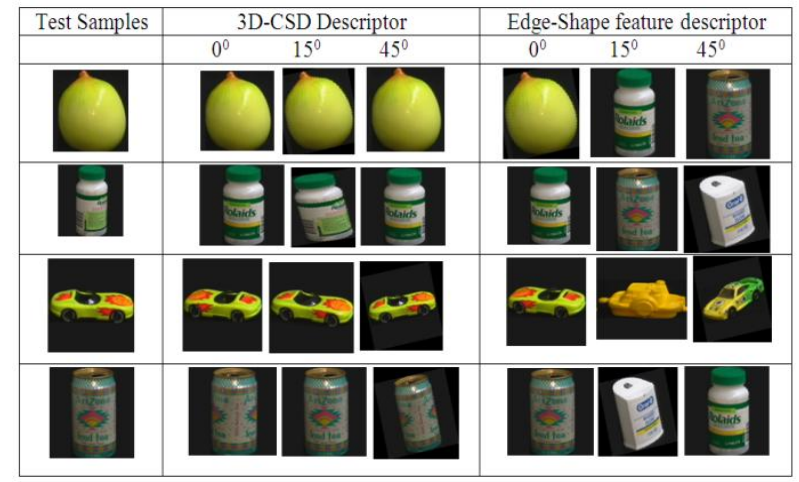

Figure 11: observations obtained for different test samples at $0,15,4^{\circ}$ orientations

\section{PERFORMANCE EVALUATION}

A process of pattern recognition based on a 3D-CSD feature descriptor for contour based feature descriptor is defined. The invariance feature for the suggested approach is derived by the depth feature which leads to more retrieval accuracy in comparison to the edge based feature descriptor approach. The retrieval accuracy of such system is observed to be improved in comparison to conventional recognition system. To evaluate the performance for such system the \% of classification accuracy for top 4 samples, and computation time is observed.

\subsection{Feature coefficient representation}

To describe the images the number of feature vectors used by the proposed 3D CSD approach and edge based approach is as shown in table 1. Here S1, S2 and S3 represents three different set of the samples passed and average number of feature vectors used to describe for each set are shown below
Table 1 Comparative representation of feature coefficient for developed approach and Edge based approach

\begin{tabular}{|c|c|c|c|}
\hline $\begin{array}{c}\text { Test } \\
\text { sample }\end{array}$ & $\begin{array}{c}\text { Contour } \\
\text { coordinates }\end{array}$ & $\begin{array}{c}\text { Depth } \\
\text { feature }\end{array}$ & $\begin{array}{c}\text { Edge } \\
\text { coordinates }\end{array}$ \\
\hline S1 & 2182 & 8 & 3082 \\
\hline S2 & 1479 & 12 & 2176 \\
\hline S3 & 2156 & 7 & 3050 \\
\hline
\end{tabular}

\subsection{True Classification}

Below table 2 shows the values obtained for the true classification

Table 2 True classification for 3DCSD and Edge Descriptor

\begin{tabular}{|c|c|c|}
\hline \multirow{2}{*}{ Test sample } & \multicolumn{2}{|c|}{ True classification (\%) } \\
\cline { 2 - 3 } & 3D-CSD & $\begin{array}{c}\text { Edge } \\
\text { descriptor }\end{array}$ \\
\hline S1 & 90 & 60 \\
\hline S2 & 92.2 & 50 \\
\hline S3 & 82.3 & 66 \\
\hline
\end{tabular}

The true classifications for the developed approaches were tested on multiple test samples and classification ratio for the top 4 retrieved sample is computed. The 3D-CSD approach results in better classification for a given test sample as in comparison to the edge descriptors. An improvement of 30\% is observed for the 3D-CSD approach, as contour and depth feature incorporation results in more effective representation. Below figure 12 shows the graph for the true classification for the proposed approach in comparison with edge based shape descriptor.

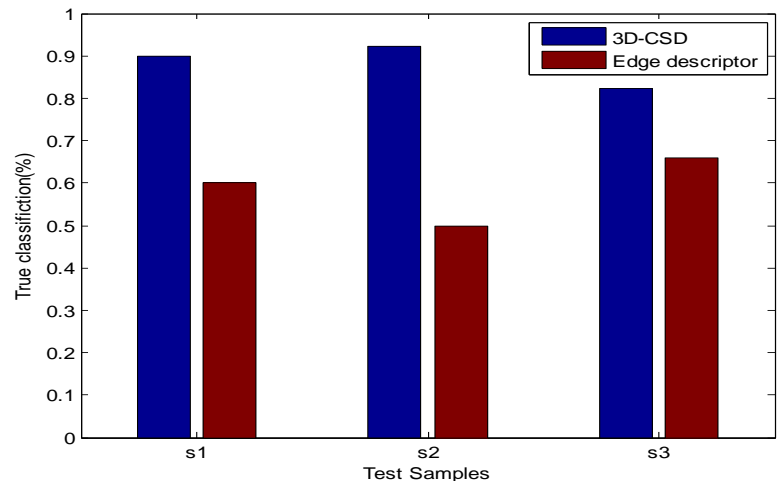

Figure 12 True classifications for the developed 3D-CSD over Edge descriptor

\subsection{Computation Time}

As contour features are used, the numbers of feature counts are minimized as illustrated in table 1 . As the feature counts are reduced, the overall processing speed is improved. This can be observed by using the below table 3 . 
Table 3 Computation time for proposed 3D CSD and Edge based feature descriptor.

\begin{tabular}{|c|c|c|}
\hline \multirow{2}{*}{ Test sample } & \multicolumn{2}{|c|}{ Computation (sec) } \\
\cline { 2 - 3 } & 3D-CSD & $\begin{array}{c}\text { Edge } \\
\text { descriptor }\end{array}$ \\
\hline S1 & 3 & 6 \\
\hline S2 & 2 & 5 \\
\hline S3 & 1.5 & 4 \\
\hline
\end{tabular}

Below figure 13 shows the computation time observed in three sets S1,S2 and S3 for the proposed approach and edge based approach.

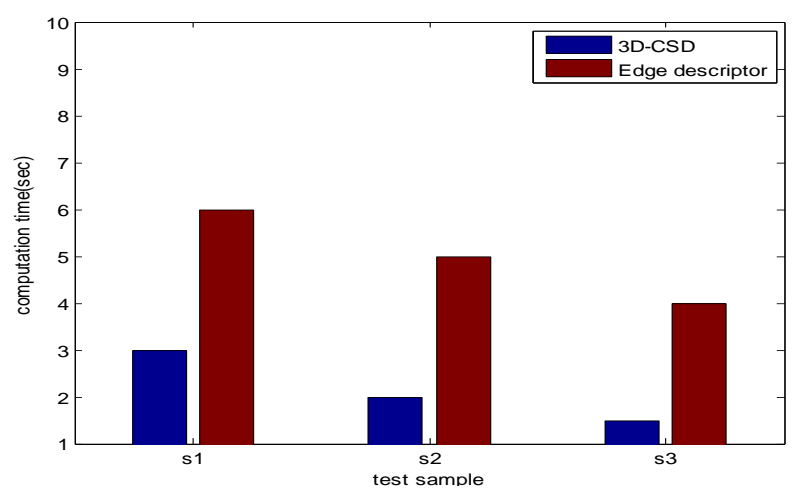

Figure 13 Computation Time plot for the developed approach in comparison with edge based approach

The comparison of the computation speed, reveals, a minimization of $3 \mathrm{sec}$ in computation time. With these observations the 3D-CSD using contour and depth feature optimizes the accuracy and processing speed of an image retrieval system.

\section{CONCLUSION}

In practical applications the images are observed in 3D representation, they are represented in an additional plane for representing depth and 3D effect. Hence, by just considering the two dimensional information leads to false classification as observed in edge based feature description. A depth parameter is also incorporated with the shape feature to represent the image feature in the proposed 3D CSD approach. Top retrieved sample in the proposed approach always matches with the query image even though the applied query image is at different orientations. The proposed approach of 3D-CSD uses less number of feature descriptor to describe the image. An improvement is also observed in true classification and the computation time. 3D CSD approach can be further investigated for applications in biomedical image processing for detecting defects in bone images. The present approach can be further improved by employing soft computing methods.

\section{REFERENCES}

[1] Novotni, M. and Klein, R., "3D Zernike Descriptors for Content Based Shape Retrieval", Proceedings of the 8th
ACM Symposium on Solid Modelling and Applications, Seattle, Washington, USA, 2003, pp. 216- 225.

[2] Bustos, B. Keim, D., Saupe, D. and Schreck,T., "Content-based 3D Object Retrieval", In IEEE Transactions on Computer Graphics and Applications, Vol. 27, No. 4, 2007, pp. 22-27.

[3] Mademlis, A., Darasb, P., Tzovarasb, D., and Strintzis, M.G., "3D Object Retrieval Using the 3D Shape Impact Descriptor", Journal of Pattern Recognition, Vol. 42 No.11, 2009, pp. 2447-2459 .

[4] Cao, L., Liu, J., and Tang, X.,"3D Object Retrieval Using 2D Line Drawing and Graph Based Relevance Feedback", Proceedings of the $14^{\text {th }}$ Annual ACM International Conference on Multimedia, Santa Barbara, CA, USA, 2006, pp. $105-108$.

[5] Ichida, H., Itoh, Y., Kitamura, Y., and Kishino, F. "Interactive Retrieval of 3D Shape Models Using Physical Objects", Proceedings of the $12^{\text {th }}$ Annual ACM International Conference on Multimedia, New York, NY, USA, 2004, pp. $692-699$.

[6] Gong, B., Xu, C., Liu, J. and Tang, X., "Boosting 3D Object Retrieval by Object Flexibility", Proceedings of the 7th ACM International Conference on Multimedia, Beijing, China, 2009, pp. 525-528.

[7] B. Bustos, D. Keim, D. Saupe, Tobias Schreck, ContentBased 3D Object Retrieval, IEEE Computer graphics and Applications, 27(4): 22- 27, 2007.

[8] Vajramushti, N., Kakadiaris, I.A., Theoharis, T., and Papaioannou, G., "Efficient 3D Object Retrieval Using Depth Images", Proceedings of the 6th ACM SIGMM International Workshop on Multimedia Information Retrieval, New York, NY, USA, 2004, pp. 189 - 196.

[9] Canny J. A Computational Approach to Edge Detection. IEEE Transactions on Pattern Analysis and Machine Intelligence. 1986; PAMI-8(6):679-698.

[10] Gonzalez R, Woods R, Eddins S. Digital Image processing using MATLAB. Upper Saddle River, N.J.: McGraw Hill Education; 2010.

[11] Dayanand Jamkhandikar, Dr. Surendra pal Singh, Dr. V.D. Mytri. Empirical Coding for Curvature Based Linear Representation in Image Retrieval System. IOSR Journal of Computer Engineering (IOSR-JCE).2015; 17(3): 5-16.

[12] Tsai, P., Shah, M.Shape from shading using linearapproximation. IVC 12.1994: 487-498

[13] S. A. Nene, S. K. Nayar, H. Murase. Columbia Object Image Library (COIL-100). Technical Report CUCS006-96. February 1996.

[14] Moon H, Chellappa R, Rosenfeld A. Optimal edge-based shape detection. IEEE Transactions on Image Processing. 2002; 11(11):1209-1227. 